The Astrophysical Journal, 247 :L105-L109, 1981 August 1

(c) 1981. The American Astronomical Society. All rights reserved. Printed in U.S.A.

\title{
RECENT COSMIC-RAY ANTIPROTON MEASUREMENTS AND ASTROPHYSICAL IMPLICATIONS
}

\author{
ANDRew Buffington ANd Stephen M. Schindler \\ California Institute of Technology \\ Received 1981 March 16; accepted 1981 A pril 14
}

\begin{abstract}
Cosmic-ray antiprotons have been detected by a new balloon-borne experiment which covers the energy range between 130 and $320 \mathrm{MeV}$. Fourteen detected events yield a measured flux of $1.7 \pm 0.5 \times 10^{-4}$ antiprotons $\mathrm{m}^{-2} \mathrm{sr}^{-1} \mathrm{~s}^{-1} \mathrm{MeV}^{-1}$. The corresponding antiproton/proton ratio is $2.2 \pm 0.6 \times 10^{-4}$, only slightly smaller than the ratio observed by other experiments at higher energies. The measured flux is significantly larger than predicted, and we discuss some cosmic-ray models which could explain this result.
\end{abstract}

Subject headings: cosmic rays: general - elementary particles - nuclear reactions

\section{INTRODUCTION}

A small flux of antiprotons $(\bar{P})$ is expected to occur in the cosmic rays as a result of the production of these particles in collisions between high energy cosmic rays, mostly protons, and the interstellar gas. A number of calculations predict a cosmic-ray $\bar{P} / P$ ratio of about $2 \times 10^{-4}$ above several GeV (Gaisser and Maurer 1973; Badhwar et al. 1975; Szabelski, Wdowczyk, and Wolfendale 1980; Stephens 1981). Golden et al. (1979) and Bogomolov et al. (1979) recently reported measurements of finite $\bar{P}$ fluxes somewhat above this expected level. In this Letter we report a new $\bar{P}$ flux measurement at low energy, a region where the secondary $\bar{P}$ spectrum is expected to drop dramatically. The integral antiproton flux inferred from the measurements is about 10 times larger than predicted under the assumptions that cosmic rays propagate according to the "leaky box" model, and that all antiprotons are secondary. Furthermore, the data show little sign of an expected kinematic suppression at low energies, a unique feature of secondary antiprotons. To accommodate the integral flux measurements, an improved model of cosmic-ray propagation would have primary protons traverse substantially more material than the $5 \mathrm{~g} \mathrm{~cm}^{-2}$ inferred from measurements of heavier cosmic rays. To fill in the low energy portion of the spectrum, stochastic, energychanging processes may have to be included in the propagation model.

\section{THE $\bar{P}$ FLUX MEASUREMENT}

The experiment detects antiprotons between about 130 and $320 \mathrm{MeV}$. It was flown by balloon from The Pas, Canada, on 1980 June 18, at an average residual atmospheric depth of $11 \mathrm{~g} \mathrm{~cm}^{-2}$, providing an exposure factor of $550 \mathrm{~m}^{2} \mathrm{sr}$. The apparatus selects $\bar{P}$ events from the larger background of normal-matter cosmic rays by combining a selective trigger with a detailed spark-chamber visualization. Figure $1 a$ shows a schematic diagram of the apparatus. The top scintillators $\left(S_{1}, S_{2}\right.$, and $\left.S_{3}\right)$ and the Cerenkov counter select events typically having an incident velocity $\leq 0.65 c$ (i.e., with $\leq 300 \mathrm{MeV}$ nucleon ${ }^{-1}$ ). The trigger requirement is $S_{1} \stackrel{\Im}{\rightarrow} S_{4}$ in coincidence, and $C$ in anticoincidence. To penetrate the $150 \mathrm{~g} \mathrm{~cm}^{-2}$ lead-plate spark chamber, thus satisfying the $S_{4}$ trigger requirement, a proton must have a kinetic energy $\gtrsim 400 \mathrm{MeV}$, which corresponds to a velocity greater than the above limit. However, antiprotons below the velocity limit can annihilate within the lead-plate chamber, with resulting daughter pions penetrating through to $S_{4}$. Once the trigger criteria are satisfied, the spark chambers are operated, giving a photographic record of the detailed event topology. The selective trigger provides an approximately 1000 -fold rejection against normal cosmicray events, with a detection efficiency of about $10 \%$ for $\bar{P}$ annihilations. An oscilloscope record of the scintillator and Cerenkov counter responses (pulse height and timing) also appears on the film.

In addition to the selective trigger, further rejection of non-antimatter background is required. The sparkchamber pictures are first selected for the multiprong topology characteristic of $\bar{P}$ annihilations. From this sample, about half of potential recorded $\bar{P}$ events are able to satisfy the final requirement imposed upon event topology, timing, and pulse height response for the scintillators. The timing permits selection of downward going particles, and the pulse height distinguishes charge $Z=1$ particles from those with $Z \geq 2$. A typical event satisfying these requirements is shown in Figures $1 b$ and $1 c$. Here a singly charged particle below Cerenkov threshold is incident from above. The particle creates a four-prong annihilation star in the leadplate chamber. Annihilations were accepted that showed three or more prongs. Seventeen events were observed in the experiment. Of these, two were discarded because of an accompanying particle which presumably assisted in meeting the trigger criterion. Estimates of residual background contamination show that an additional event must be subtracted to remove this final residue. Based on Monte Carlo calculations, the effective instrument geometry factor, including all trigger and identification criteria, is $0.012 \mathrm{~m}^{2} \mathrm{sr}$. Additional details about the apparatus, calibration, balloon- 


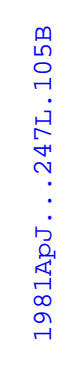

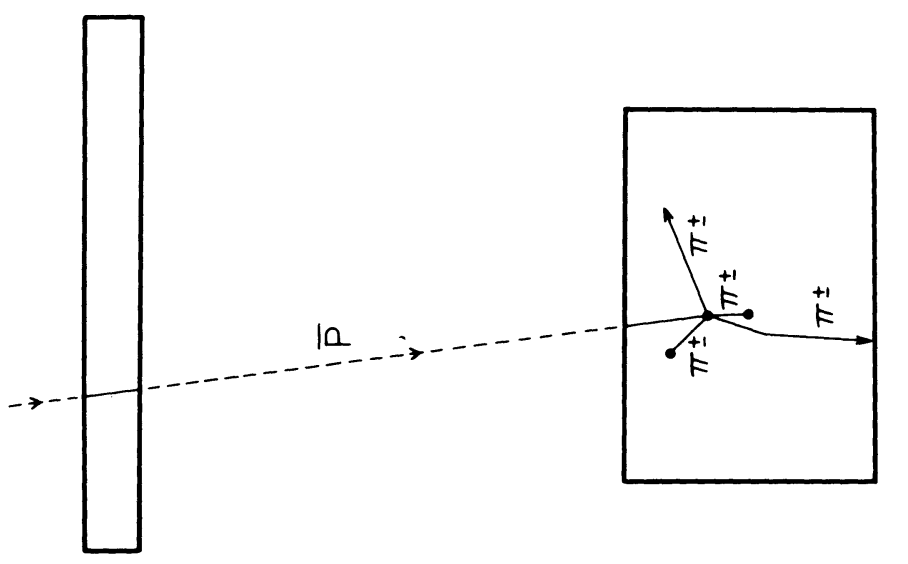

$\dot{0}$
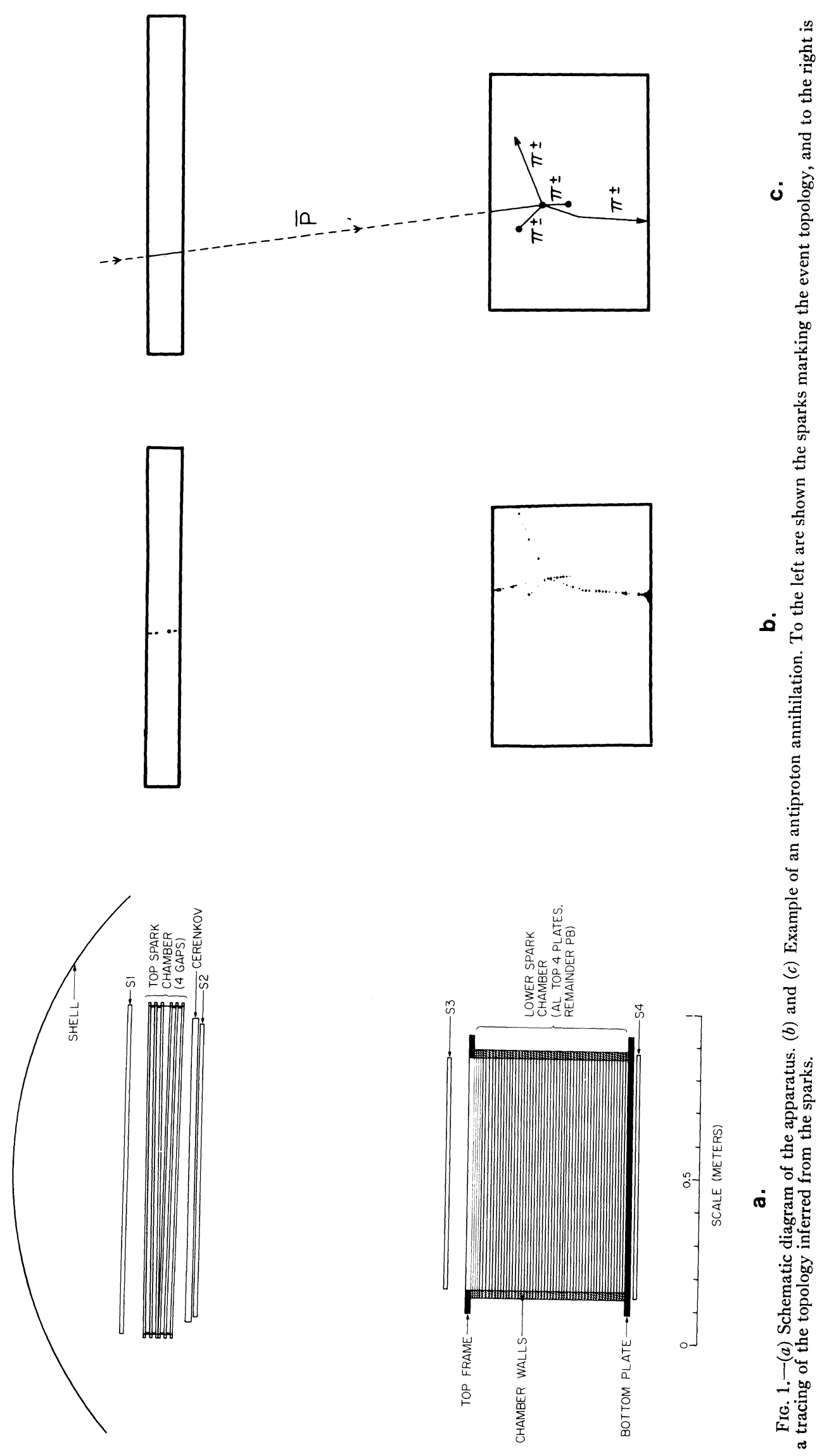
flight exposure, and data analysis appear elsewhere (Buffington, Schindler, and Pennypacker 1981).

\section{RESULTS}

After correction for interaction losses in the atmosphere and apparatus, 14 observed antiprotons yield a differential flux of $1.7 \pm 0.5 \times 10^{-4}$ antiprotons $\mathrm{m}^{-2} \mathrm{sr}^{-1}$ $\mathrm{s}^{-1} \mathrm{MeV}^{-1}$ ) at the top of the atmosphere. The flux of atmospherically produced antiprotons is negligible at this energy (Gaisser and Levy 1974; Buffington et al. 1979; Buffington, Schindler, and Pennypacker 1981). The experiment did not provide an accurate measurement of the primary proton flux because of atmospheric secondary protons. To form a $\bar{P} / P$ ratio, we use results based on an extrapolation of IMP 8 satellite proton flux measurements taken just before the balloon flight (see Buffington, Schindler, and Pennypacker 1981). This procedure yields a proton flux of 0.8 protons $\mathrm{m}^{-2} \mathrm{sr}^{-1} \mathrm{~s}^{-1} \mathrm{MeV}^{-1}$ in our energy range. The corresponding $\bar{P} / P$ ratio is $2.2 \pm 0.6 \times 10^{-4}$, somewhat smaller than the ratios observed by other experiments at higher energy (Golden et al. 1979; Bogomolov et al. 1979). One should note that the correct ratio may be up to a factor of 2 larger, since the $I M P \&$ proton flux at this time was larger than was observed at the corresponding period in the previous solar cycle.

Figures 2 and 3 show the measured $\bar{P}$ fluxes and $\bar{P} / P$ ratios, together with calculations assuming these particles are entirely of secondary origin and propagate according to the leaky box model. The Gaisser and Levy (1974) and Gaisser and Maurer (1973) calculations do not include a factor of $\sim 1.34$ to take account of heavy nucleus parents of antiprotons. The calculated fluxes apply to interstellar space, while the measurements are at $1 \mathrm{AU}$. Solar modulation affects the spectral shape, especially at low energy. Figure 2 also shows the calculation of Gaisser and Levy (1974) for the expected change in their $\bar{P}$ spectrum as a result of solar modulation for 1969, which corresponds to the period of interest in the current solar cycle. A similar alteration in spectral shape can be applied to the other calculations. Alternatively, in Figure 3, one could move our $\bar{P} / P$ data point to the right by about $600 \mathrm{MeV}$ to get an appropriate estimate for interstellar space (Urch and Gleeson 1973).

\section{DISCUSSION}

It would appear that the three $\bar{P}$ measurements agree fairly well with the calculation of Badhwar et al. (1975). However, Stephens (1981) has shown that the discrepancy between his calculation and that of Badhwar et al. could result from the parameterization of the interaction dynamics; Badhwar et al. use values which yield substantially larger $\bar{P}$ production at lower parent-proton energies than is observed in accelerator measurements. This results in Badhwar et al. predicting a much larger flux near $1 \mathrm{GeV}$. Thus, the lower curves in Figures 2 and 3 probably represent the best presentday estimate of the $\bar{P}$ flux within the leaky box model for cosmic rays in the Galaxy, with the agreement with Badhwar et al. being fortuitous.
It is clear that the correspondence is poor between the three experimental results and the remaining calculations. Drawing a reasonable smooth curve through the $\bar{P}$ differential flux measurements yields an integral flux about 10 times larger than predicted by theory. Figure 3 shows that the measured $\bar{P}$ spectrum is consistent with having the same shape as the proton spectrum, but reduced in intensity by a factor of about $3 \times 10^{-4}$. The low energy measurement reported here is about 1000 times higher than the prediction of Stephens (1981), although solar modulation may reduce this difference by a factor of $\sim 10$.

Table 1 provides a list of possibilities that could resolve the discrepancy between measurement and theory. We feel that changing the assumptions about secondary antiprotons represents a preferable course of action, compared to suggesting the existence of primary antiprotons.

As shown in Table 1, primary antiprotons might be left over from the big bang, might have been generated in regions of bulk antimatter nearby, or might be produced by some exotic process which does not create

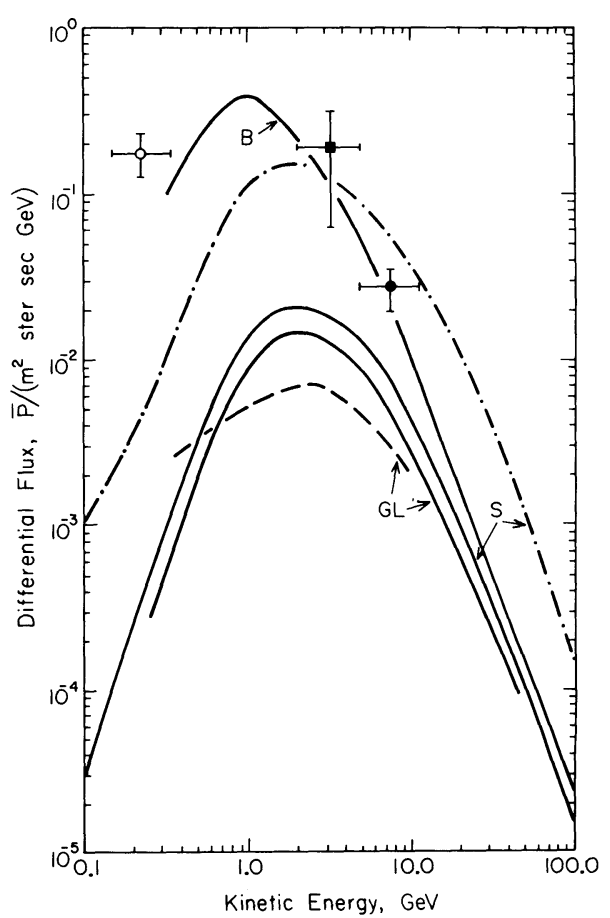

Fig. 2.-A plot of measured and calculated antiproton differential fluxes as a function of energy. (\$) Golden et al. (1979); (\$) Bogomolov et al. (1979); ( $\Phi)$ this experiment (Buffington, Schindler, and Pennypacker 1981). We note that the Bogomolov et al. measurement is based on only two observed $\bar{P}$ 's. The curve labeled $B$ is from Badhwar et al. (1975), multipled by $19000 E_{\mathrm{tot}}-2.75$ to remove the proton flux denominator from the $\bar{P} / P$ ratio. The experimentally measured fluxes have been similarly derived from reported $\bar{P} / P$ ratios. The curves labeled $S$ are from Stephens (1981); the lower one is for the leaky box model and the upper one for the closed galaxy model. The curves labeled $G L$ are from Gaisser and Levy (1974); the upper one is for the leaky box model and the lower one includes the effects of solar modulation. 
heavier antimatter. Antimatter left over from the big bang must have spent the bulk of the time since then in regions of low density, or it would have all been annihilated. The questions of extragalactic storage or antimatter galaxies remain open (Senjanovic and Stecker 1980). However, at the energies of interest here, a means would have to be provided for the antimatter to enter the Galaxy and reach us in the form of cosmic rays. Alternatively, antiprotons might reach us from galactic antimatter regions, a topic recently reviewed by Steigman (1976). If so, the observed absence of antihelium and heavier antimatter in the cosmic rays must be explained (Stephens 1981). Antimatter cosmic rays would be expected to have a similar elemental composition and spectral shape to normal-matter cosmic rays, if they were formed in antimatter regions similar to normal-matter ones. However, as a secondary objective, the experiment being reported here placed a $95 \%$ confidence level upper limit to the antihelium/ helium ratio of $2.2 \times 10^{-5}$, a decade lower than the measured $\bar{P} / P$ ratio. Other experiments have observed similar small upper limits for this ratio and the corresponding ratio for heavier elements (Steigman 1976). One can speculate that the heavier antinuclei were destroyed by passage from the regions of antimatter through a thick slab of material which annihilates most of the heavier antinuclei, but only a fraction of the antiprotons. Such a slab would have to be everywhere thicker than about $20 \mathrm{~g} \mathrm{~cm}^{-2}$. On the other hand, the assumption of equal $\overline{\mathrm{He}} / \bar{P}$ and $\mathrm{He} / P$ ratios might not be valid. However, we feel a strong claim of primary antimatter, with its attendant impact on cosmology, would probably require a discovery of antinuclei heavier than antiprotons, because of the possible explanation for these latter particles as secondaries.

Table 1 indicates two hypotheses involving only secondary processes which together could explain the observations. A large integral $\bar{P}$ flux would result if parent protons traversed substantially more than the $5 \mathrm{~g} \mathrm{~cm}^{-2}$ of interstellar material inferred from measurements of heavier nuclei. There are a number of models which could satisfy this requirement; all of these have a significantly different history for protons compared with the heavier nuclei. Rasmussen and Peters (1975) and Peters and Westergaard (1977) have discussed a "closed galaxy" model in which cosmic rays are confined in the Galaxy by large-scale magnetic fields, with the resulting fluxes determined through a balance between creation and destruction by interactions. In this model most of the heavier nuclei are "young" and come from relatively nearby sources along the galactic spiral arms. Thus, these particles have passed through only

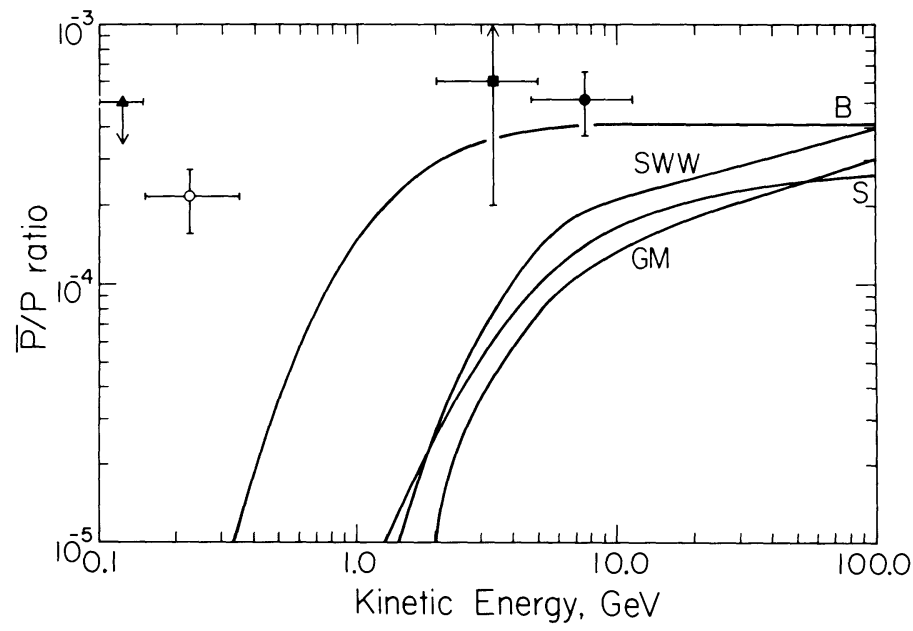

FIG. 3.-A plot of measured and calculated $\bar{P} / P$ ratios as a function of energy. The data points are as in Fig. 2, except ( $\Psi$ ) is deduced as a $1 \sigma$ upper limit from Apparao (1968), in which no events were seen in about 3000 stopping protons. The curves are $B$ : Badhwar et al. (1975); $S W W$ : Szabelski, Wdowczyk, and Wolfendale (1980); GM: Gaisser and Maurer (1973); and $S$ : Stephens (1981). Correction for the effect of solar modulation is expected to move the data points to the right. For this experiment the amount is about $600 \mathrm{MeV}$.

TABLE 1

Possible Alterations to Calculation of Expected Antiproton Flux

\begin{tabular}{lll}
\hline & Assumption & Comment \\
\hline $\begin{array}{l}\text { Primary } \\
\text { Antiprotons }\end{array}$ & $\left\{\begin{array}{l}\text { a. from big bang } \\
\text { b. from antimatter regions } \\
\text { c. exotic production processes } \\
\begin{array}{l}\text { Antiprotons } \\
\text { Andary }\end{array}\end{array}\right.$ & $\begin{array}{l}\text { Problem with storage/injection. } \\
\text { Spectra and elemental composition might be similar to those of common matter. } \\
\text { Antiprotons produced with no heavier antimatter. }\end{array}$ \\
$\begin{array}{ll}\text { a. increase material traversed } \\
\text { b. stochastic processes }\end{array}$ & $\begin{array}{l}\text { Increases expected integral antiproton flux. } \\
\text { Alters antiproton spectrum shape. }\end{array}$ \\
\hline
\end{tabular}


about $5 \mathrm{~g} \mathrm{~cm}^{-2}$, as deduced from measurements of cosmic-ray $\mathrm{Li}, \mathrm{Be}, \mathrm{B}$, and the subiron elements. However, a substantial fraction of the protons are "old" cosmic rays, and have passed through up to 10 times more of the material than the young component. Stephens (1981) has developed this model to produce a larger $\bar{P}$ flux, which fits the data of Golden et al. (1979). Figure 2 shows his calculation. The increased integral flux of antiprotons for this model approximately matches that inferred from the $\bar{P}$ measurements, although the spectral shape is not correct. Questions have been raised (Badhwar and Stephens 1976) about the compatibility of the closed galaxy model with positron and radio spectrum measurements. If these difficulties can be overcome, the closed galaxy model provides a means of generating a larger $\bar{P}$ flux without contradicting the heavier nucleus cosmic-ray measurements.

Even if a larger integral $\bar{P}$ flux can be justified, a mechanism is still required to fill in the low energy portion of the $\bar{P}$ spectrum. Szabelski, Wdowczyk, and Wolfendale (1980) suggest that stochastic, energychanging interactions during propagation could have this effect. While no calculations have been performed in this context, there has been a continuing interest in interstellar stochastic processes since Fermi's (1949) original suggestion that particle collisions with magnetic clouds could accelerate cosmic rays. Cowsik (1980) has shown that such processes cannot be responsible for the spectral shape of the heavy nuclei, since in this case the secondary nuclei should have a flatter spectrum than primary nuclei, contrary to the observations. Cowsik's analysis, however, does not exclude the possibility that stochastic processes are important for a secondary $\bar{P}$ flux, which may be significantly older than the fluxes of the heavier nuclei.

In summary, a primary antiproton hypothesis cannot be ruled out. If the antiprotons are viewed as being entirely secondaries, they could be of key importance for determining the history of cosmic-ray protons. In particular, the recent $\bar{P}$ measurements would suggest a substantially different history for protons compared with the medium or heavy elements.

This work was supported by grants NGR 05-003-553 and NGR 05-002-160 from the National Aeronautics and Space Administration. C. R. Pennypacker provided invaluable assistance in the preparation of the experiment.

\section{REFERENCES}

Apparao, M. V. K. 1968, Proc. 10th Internat. Cosmic Ray Conference, Calgary, (Can.J. Phys., 46, S654).

Badhwar, G. D., Golden, R. L., Brown, M. L., and Lacy, J. L. 1975, A p. Space Sci., 37, 283.

Badhwar, G. D., and Stephens, S. A. 1976, Phys. Rev. D, 14, 356.

Bogomolov, E. A., Lubyanaya, N. D., Romanov, V. A., Stepanov, S. V., and Shulakova, M. S. 1979, Proc. 16th Internat. Cosmic Ray Conference, Kyoto, 1, 330.

Buffington, A., Pennypacker, C. R., Lubin, P. M., and Smoot, G. F. 1979, Proc. 16th Internat. Cosmic Ray Conference, Kyoto, $1,337$.

Buffington, A., Schindler, S. M., and Pennypacker, C. R. 1981, $A$ p. J., submitted.

Cowsik, R. 1980, Ap. J., 241, 1195.

Fermi, E. 1949, Phys. Rev., 75, 1169.
Gaisser, T. K., and Levy, E. H. 1974, Phys. Rev. D, 10, 1731. Gaisser, T. K., and Maurer, R. H. 1973, Phys. Rev. Letters, 30, 1264.

Golden, R. L., Horan, S., Mauger, B. G., Badhwar, G. D., Lacy, J. L., Stephans, S. A., Daniel, R. R., and Zipse, J. E. 1979, Phys. Rev. Letters, 43, 1196

Peters, B., and Westergaard, N. J. 1977, Ap. Space Sci., 48, 21.

Rasmussen, I. L., and Peters, B. 1975, Nature, 258, 412.

Senjanović, G., and Stecker, F. W. 1980, Phys. Letters, 96B, 285.

Steigman, G. A. 1976, Ann. Rev. Astr. Ap., 14, 339.

Stephens, S. A. 1981, Ap. Space Sci., 76, 87.

Szabelski, J., Wdowczyk, J., and Wolfendale, A. W. 1980, Nature, $285,386$.

Urch, I. H., and Gleeson, L. J. 1973, Ap. Space Sci., 20, 177.

Andrew Buffington and Stephen M. Schindler: 220-47 Downs Laboratory, California Institute of Technology, Pasadena, CA 91125 